\title{
Néphrotoxicité du lithium ${ }^{\star}$ Renal toxicity of lithium
}

\author{
Aude Servais \\ Service de néphrologie adulte, hôpital Necker, université Paris Descartes, 149 rue de Sèvres, \\ 75015 Paris, France \\ ${ }^{*}$ Auteur correspondant \\ e-mail : aude.servais@aphp.fr
}

${ }^{\star}$ Cet article est paru initialement dans EMC - Néphrologie 2015;12(4):1-6 [article 18-066-D-

20]. Nous remercions la rédaction d'EMC-Néphrologie pour son aimable autorisation de reproduction.

\begin{abstract}
Résumé
Les sels de lithium restent le traitement de référence du trouble bipolaire, mais leur utilisation se heurte à un indice thérapeutique étroit et à des effets secondaires. Deux types de néphrotoxicité sont observés: un trouble de la concentration des urines, précoce, dès huit semaines de traitement, et une réduction du débit de filtration glomérulaire à long terme. Le diabète insipide néphrogénique est observé chez 40 à $50 \%$ des patients. Cette réduction de la capacité de concentration des urines est corrélée à la durée d'exposition au lithium. Ce trouble de concentration des urines se traduit par un syndrome polyuropolydipsique qui peut être chiffré jusqu'à $10 \mathrm{~L} / \mathrm{j}$. II serait moins fréquent en cas de prise unique quotidienne. Chez les patients, il apparaît en général après huit semaines de traitement et, après l'arrêt du traitement, les symptômes régressent lentement. Ils sont cependant inconstamment réversibles, surtout si le traitement a été prolongé plus de 15 ans. Une insuffisance rénale chronique est observée chez certains patients traités depuis 10 à 20 ans. Sa prévalence est chiffrée à $12 \%$ après 19 ans de traitement. Certains patients $(0,5 \%)$ sont susceptibles d'évoluer vers l'insuffisance rénale terminale. Le seul facteur de risque retrouvé est la durée d'exposition au lithium. II s'agit d'une néphropathie tubulo-interstitielle chronique dont l'évolution est le plus souvent lente. Des kystes sont fréquemment visualisés sur les examens d'imagerie. La question de l'arrêt du traitement est une question délicate. Le néphrologue doit prendre en compte la gravité éventuelle de la maladie psychiatrique qui peut être associée à un risque élevé de récidive et de suicide. La discussion doit donc se faire de manière conjointe entre le patient, le néphrologue et le psychiatre. Le bénéfice rénal de l'arrêt du traitement n'est réel que si l'insuffisance rénale est modérée (débit de filtration glomérulaire supérieur à $40 \mathrm{~mL} / \mathrm{min}$ ).
\end{abstract}

\section{Mots-clés}

diabète insipide néphrogénique ; kyste ; lithium ; néphropathie tubulo-interstitielle chronique ; syndrome polyuropolydipsique ; trouble bipolaire

\begin{abstract}
Besides its efficiency, lithium has a narrow therapeutic index and can result in considerable toxicity. Among the potential side effects, two types of renal toxicity are observed: a decreased renal concentrating ability and a chronic renal failure. Lithium-induced polyuria is frequent, estimated to affect up to $40 \%$ of patients, and develops usually early. It may be irreversible, especially if the treatment has been prescribed for more than 15 years. A chronic renal failure is observed in patients treated for more than 10 to 20 years. Its prevalence is estimated at $12 \%$ after 19 years of treatment. Some patients $(0.5 \%)$ may reach end stage
\end{abstract}


renal disease. The major risk factor is the duration of exposure to lithium. Discussion about stopping or not lithium in case of renal failure needs multidisciplinary expertise and depends on psychiatric status and renal function.

\section{Keywords}

bipolar disorder; chronic tubulointerstitial nephropathy; cyst; lithium; nephrogenic diabetes insipidus; polyuropolydipsic syndrome

\section{Introduction}

Les sels de lithium restent le traitement de référence du trouble bipolaire, maladie psychiatrique fréquente se traduisant par l'alternance d'épisodes dépressifs sévères et d'épisodes maniaques. Même s'ils sont utilisés depuis plus de 50 ans, les preuves les plus convaincantes de leur efficacité à long terme proviennent d'essais cliniques randomisés plus récents dans lesquels ils tenaient le rôle de comparateur ${ }^{[1,2]}$. Une méta-analyse de cinq études contrôlées où le lithium était utilisé en traitement d'entretien contre placebo a montré qu'il diminue le risque de récidive maniaque de $38 \%$ et de récidive dépressive de $28 \%$. II s'agit du seul traitement ayant prouvé une réduction du risque de suicide de plus de $50 \%{ }^{[3]}$. Cependant, ces bénéfices psychiatriques sont contrebalancés par son indice thérapeutique étroit et ses effets secondaires.

\section{Physiologie}

II s'agit d'un cation monovalent dont le comportement est similaire à celui du sodium et qui est filtré librement à travers le glomérule. Soixante-quinze pour cent de la charge filtrée sont réabsorbés au niveau du tubule proximal par voie paracellulaire. Les réabsorptions du lithium et du sodium sont proportionnelles. Une petite fraction est réabsorbée au niveau distal dans le canal collecteur, à travers le canal sodium épithélial $(\mathrm{ENaC})^{[4]}$ (Fig. 1). En cas de déplétion en sel, $20 \%$ sont réabsorbés au niveau de la branche ascendante large de l'anse de Henle. La concentration sérique de lithium augmente donc chez les patients traités par lithium lorsque le débit de filtration glomérulaire est diminué, en cas d'hypovolémie, de prise d'antiinflammatoires non stéroïdiens, de traitement par inhibiteur de l'enzyme de conversion ou par antagoniste du récepteur de l'angiotensine 2 , ou quand la réabsorption est stimulée par un régime désodé ou des diurétiques ${ }^{[5]}$.

Plusieurs types de néphrotoxicité peuvent être observés ${ }^{[6]}$. D'une part, un trouble de la concentration des urines peut être retrouvé, précocement, dès huit semaines de traitement ${ }^{[7]}$. D'autre part, une réduction du débit de filtration glomérulaire peut survenir à long terme, après 10 à 20 ans de traitement ${ }^{[8-10]}$. En marge de ces complications rénales, une hypercalcémie est fréquemment mise en évidence.

\section{Diabète insipide néphrogénique}

Un diabète insipide néphrogénique est observé chez 40 à $50 \%$ des patients. Cette réduction de la capacité de concentration des urines est corrélée à la durée d'exposition au lithium [11]. Cependant, un seul mois de traitement chez des volontaires sains réduit déjà l'osmolalité urinaire et l'excrétion d'aquaporine $2^{[12]}$. Ce trouble de concentration des urines se traduit par un syndrome polyuropolydipsique qui peut être chiffré jusqu'à $10 \mathrm{~L} / \mathrm{j}$. II serait moins fréquent en cas de prise unique quotidienne ${ }^{[13]}$. Chez les patients, il peut apparaître précocement, dès huit semaines de traitement ${ }^{[7]}$. Après l'arrêt du traitement, les symptômes régressent lentement, jusqu'à huit semaines après ${ }^{[14]}$. Ils sont cependant inconstamment réversibles, surtout si le traitement a été prolongé plus de 15 ans ${ }^{[15]}$. 
Cet accroissement de la diurèse chez les patients traités par lithium est dû à une régulation négative et à une diminution du transfert vers la membrane des canaux aquaporine 2 (AQP2) ${ }^{[4,16]}$. Cela a été montré initialement dans des modèles murins. L'AQP3 est également régulée négativement par le traitement par lithium ${ }^{[17]}$. Le lithium engendre donc une forme de diabète insipide ne répondant pas à la vasopressine par régulation négative de l'expression de l'AQP2 sur la membrane apicale et de l'AQP3 sur la membrane basolatérale des cellules principales du canal collecteur ${ }^{[4]}$ (Fig. 1).

II s'y associe une dérégulation de l'expression de ENaC dans la partie corticale et médullaire externe du canal collecteur. En effet, le lithium entre dans les cellules principales du canal collecteur au niveau de la membrane apicale par le canal ENaC (Fig. 1). Le sodium est exporté de la cellule vers le sang par la $\mathrm{Na}^{+}, \mathrm{K}^{+}$-ATPase située sur la membrane basolatérale, mais le lithium est un mauvais substrat de cette pompe et s'accumule dans la cellule. Un certain nombre de voies intracellulaires ont ensuite été impliquées. L'altération de la voie de la protéine kinase A semble être l'un des principaux mécanismes d'action du lithium. Le lithium inhibe l'activité de l'adénylate cyclase à la fois basale et stimulée par le 1-deamino-8$D$-arginine vasopressin (dDAVP), réduisant de ce fait la production d'adénosine monophosphate $3^{\prime}-5^{\prime}$ cyclique (AMPc). Par ailleurs, la régulation de l'adénylate cyclase dépend aussi de la glycogène synthase kinase $3 \beta$ (GSK3 $\beta)^{[18]}$.

Parallèlement aux voies dépendant de l'AMPc, d'autres enzymes clés sont régulées par le lithium. II induit une phosphorylation d'Akt, ce qui engendre une inhibition de GSK3 $\beta$ via un mécanisme de phosphorylation ${ }^{[19]}$. L'inhibition de l'activité de GSK3 $\beta$ est associée à une accumulation de $\beta$-caténine dans les cellules principales. Cette protéine est impliquée dans l'adhésion cellulaire et joue un rôle de corégulateur transcriptionnel de gènes de croissance cellulaire. Cette voie de régulation pourrait conduire au taux de prolifération élevé des cellules principales observé sous traitement par lithium ${ }^{[20]}$.

Le diabète insipide induit par le lithium implique aussi les cellules interstitielles médullaires. Le lithium inhibe GSK3 $\beta$ et augmente l'expression de cyclo-oxygénase 2 (COX-2) dans les cellules interstitielles médullaires. II s'y associe une augmentation de l'excrétion urinaire de prostaglandine $E_{2}\left(P E_{2}\right)$ et un effet diurétique ${ }^{[21]}$. En outre, le lithium réduit l'abondance des transporteurs de l'urée UT-A1 et UT-B, diminuant ainsi l'osmolalité interstitielle de la médullaire et donc le gradient nécessaire à une réabsorption efficace de l'eau ${ }^{\text {[22] }}$. La composition cellulaire du canal collecteur est remaniée par le lithium. En effet, on observe une modification de la distribution cellulaire avec augmentation de la proportion des cellules intercalaires aux dépens des cellules principales.

Chez l'homme, Bedford et al. ont montré qu'il existait une diminution de l'excrétion urinaire d'AMPc et d'AQP2 qui est corrélée à la durée d'exposition au lithium ${ }^{[11]}$. En outre, la capacité de réponse au dDAVP est diminuée. En cas de diabète insipide néphrogénique invalidant, l'utilisation d'amiloride a été proposée pour inhiber le canal $\mathrm{ENaC}$ et, de ce fait, bloquer l'entrée du sodium et du lithium dans la cellule principale ${ }^{[23]}$. Dans une étude en cross-over réalisée chez des patients recevant du lithium, Bedford et al. ont administré un traitement par $10 \mathrm{mg}$ d'amiloride par jour pendant 6 semaines et observé une augmentation de l'osmolalité urinaire ${ }^{[11]}$. Dans cette étude, la lithiémie est restée stable, mais il convient de surveiller la kaliémie et la fonction rénale. Cependant, le syndrome polyuropolydipsique est en général bien toléré par les patients et le recours à un traitement est exceptionnel. Ces patients ne doivent pas être mis au régime désodé, ni en restriction hydrique. 


\section{Mise au point}

- Le syndrome polyuropolydipsique est dû à une régulation négative et à une diminution du transfert vers la membrane des canaux AQP2.

- Il s'y associe une dérégulation de l'expression de ENaC dans la partie corticale et médullaire externe du canal collecteur.

Le lithium affecte également le système rénine-angiotensine : il semble causer une résistance à l'aldostérone au niveau du canal collecteur, ce qui peut être un élément important de la natriurèse qui accompagne souvent la polyurie ${ }^{[24]}$. En outre, les interactions entre le lithium et les inhibiteurs du système rénine-angiotensine peuvent être cliniquement dangereuses puisque la contraction volémique peut engendrer une plus grande rétention du lithium.

\section{Hypercalcémie et hyperparathyroïdie}

Le traitement par lithium au long cours peut aussi être associé à une hypercalcémie et une hyperparathyroïdie. La prévalence de l'hypercalcémie est très variable selon les études, entre 10 et $42 \%$ des patients ${ }^{[9,25-28]}$. Cependant, la prévalence de l'hyperparathyroïdie n'est estimée qu'entre 10 et $15 \%{ }^{[29]}$. Alors que la prévalence de l'hyperparathyroïdie dans la population générale est d'environ $0,5 \%$, elle est néanmoins presque 7,5 fois supérieure dans une population exposée au lithium pendant plus de 15 ans ${ }^{[27]}$. En général, l'hyperparathyroïdie et l'hypercalcémie compliquant un traitement par lithium sont modérées. Des cas d'hypercalcémie sévère conduisant au coma ont toutefois été décrits ${ }^{[30]}$. Une «fausse » hypercalcémie due à une déplétion du volume plasmatique en lien avec le diabète insipide néphrogénique doit être éliminée en premier lieu. La prévalence de cette hypercalcémie induite par le lithium est plus importante chez les insuffisants rénaux chez lesquels la calciurie est réduite ${ }^{[9]}$. Chez certains patients, des complications à types de lithiase, néphrocalcinose ou ostéoporose sont observées ${ }^{[29,31-33]}$. Même si une sécrétion anormale de parathormone apparaît in vitro après une courte exposition au lithium ${ }^{[34]}$, l'incidence clinique de l'hyperparathyroïdie n'est augmentée significativement qu'après 15 ans d'exposition ${ }^{[27,31]}$. Cette association entre prise de lithium et hyperparathyroïdie a plusieurs explications possibles. Elle pourrait résulter d'une altération du récepteur sensible au calcium avec un déplacement vers la droite de la courbe de réponse au calcium de la parathormone ${ }^{[35]}$. II en résulterait un rétrocontrôle sur la parathormone à un seuil de calcémie plus élevé ${ }^{[36,37]}$. Le lithium pourrait aussi promouvoir la prolifération de tissu parathyroïdien anormal ${ }^{[38,39]}$.

\section{Néphropathie induite par le lithium}

Une insuffisance rénale chronique est observée chez certains patients traités depuis 10 à 20 ans. Le nombre maximal de nouveaux cas d'insuffisance rénale chronique se situait entre 16 et 20 ans de traitement dans l'étude d'une cohorte de 114 patients traités par lithium ${ }^{\text {[40]. }}$. Presne et al. ont bien montré une corrélation entre la clairance de créatinine et la durée du traitement par lithium ${ }^{[9]}$. Dans la mesure où les troubles bipolaires sont susceptibles de commencer à un jeune âge (15-25 ans), conduisant alors à la prescription de lithium, il existe un risque d'insuffisance rénale chronique dès l'âge de 40 ans.

\subsection{Prévalence}

L'analyse de la fonction rénale dans une vaste cohorte de patients traités par lithium a montré que $4 \%$ des patients avaient une créatinine augmentée après 6,5 ans de traitement et $12 \%$ après 19 ans de traitement ${ }^{[41]}$. Quelques années plus tard, la même équipe a montré que la 
prévalence des patients traités par lithium et ayant une créatinine supérieure à $150 \mu \mathrm{mol} / \mathrm{L}$ était de 1,2\%, insuffisance rénale terminale exclue. De même, dans la cohorte de Lepkifker et al., chez des patients traités par lithium depuis 16,8 ans en moyenne, $21 \%$ avaient une créatinine anormale, supérieure à $133 \mu \mathrm{mol} / \mathrm{L}^{[40]}$. Ces études ne rapportaient pas de calcul du débit de filtration glomérulaire.

Bassilios et al. ont utilisé une base de données de laboratoires de ville afin d'analyser la fonction rénale de patients ayant un dosage concomitant de lithiémie ${ }^{[42]}$. Un débit de filtration glomérulaire estimé selon la formule Modification of the diet in renal disease (MDRD) inférieur à $60 \mathrm{~mL} / \mathrm{min} / 1,73 \mathrm{~m}^{2}$ était observé chez $39 \%$ des patients ayant un âge compris entre 20 et 39 ans et chez $85 \%$ des patients âgés de plus 70 ans. Ces chiffres étaient clairement plus élevés que ceux retrouvés dans des cohortes de patients issus de la population générale. La prévalence d'un débit de filtration glomérulaire bas augmentait avec l'âge, mais était très élevée dans le groupe de patients les plus jeunes. Les lithiémies étaient équivalentes dans les différents groupes d'âge.

Récemment, Minay et al. ont comparé une population de patients recevant du lithium à un groupe contrôle et ont montré que dans $17 \%$ des cas, le débit de filtration glomérulaire estimé était inférieur à $60 \mathrm{~mL} / \mathrm{min} / 1,73 \mathrm{~m}^{2}$, ce qui était significativement plus que chez les contrôles $(13 \%){ }^{[43]}$. Cependant, les auteurs signalaient que $31 \%$ des patients n'avaient pas de dosage de la créatinine au cours des 2 années de l'étude.

Une méta-analyse d'études de cas-témoins comparant le débit de filtration glomérulaire estimé de patients sous lithium à celui de témoins a montré une réduction du débit de $5 \mathrm{~mL} / \mathrm{min}$ chez les patients traités, mais pour une période d'observation d'un an seulement, ce qui ne répond pas à la question de la toxicité rénale à long terme ${ }^{[44]}$.

\subsection{Insuffisance rénale terminale}

Certains patients sont susceptibles d'évoluer vers l'insuffisance rénale terminale: 18 sur 3369 dans l'étude de Bendz et al., soit $0,5 \%{ }^{[8]}$. Cette prévalence était six fois supérieure à celle de la population générale. Les patients avaient plus de 46 ans lorsqu'ils arrivaient au stade de la dialyse, ils avaient été traités en moyenne 23 ans et avaient arrêté le lithium depuis plus de 10 ans pour plus de la moitié d'entre eux. Parmi les dialysés en France, la prévalence de la néphropathie au lithium a été estimée à $0,22 \%{ }^{[9]}$. À l'hôpital Necker à Paris, entre 1989 et 2000, deux patients sur 1391 mis en dialyse, soit 0,14\% des patients incidents, avaient une néphropathie au lithium. Dans les registres australiens et néozélandais, l'incidence de l'insuffisance rénale terminale induite par le lithium se situe entre 0,2 et 0,7\% en 2000-2003. Dans l'étude de Presne et al., le seul facteur de risque retrouvé d'évoluer vers le stade terminal de l'insuffisance rénale était le débit de filtration glomérulaire initial, comme attendu. L'arrêt du traitement et les lithiémies moyennes n'avaient en revanche pas d'impact sur ce risque évolutif. Entre l'âge de début du traitement et la mise en dialyse, il existait un intervalle d'environ 20 ans.

\subsection{Présentation clinique}

II s'agit d'une néphropathie le plus souvent asymptomatique, sans hypertension artérielle ni protéinurie, sauf à un stade avancé. La présence d'un syndrome néphrotique est rare : seuls 26 cas ont été rapportés ${ }^{[10]}$. Un trouble de la concentration des urines est souvent associé. Le seul facteur de risque retrouvé est la durée d'exposition au lithium. L'existence de comorbidités peut également jouer un rôle dans la progression de la néphropathie. Les éventuels épisodes d'intoxication au lithium ne semblent pas être déterminants. En revanche, le nombre de prises pourrait influencer la néphrotoxicité avec une tolérance meilleure en cas de prise unique. 


\subsection{Diagnostic}

Le diagnostic repose sur un faisceau d'arguments associant une prise de lithium prolongée, actuelle ou ancienne, de plus de 10 ans, un tableau de néphropathie tubulo-interstitielle chronique, sans autre cause. L'évolution est le plus souvent lente avec un déclin moyen du débit de filtration glomérulaire de $2,2 \mathrm{~mL} / \mathrm{min} / \mathrm{an}^{[45]}$. L'imagerie (échographie ou imagerie par résonance magnétique [IRM]) met en évidence des microkystes corticaux et médullaires au sein de reins de taille conservée dans 33 à $62,5 \%$ des cas ${ }^{[10,46]}$. Ces kystes sont issus du tubule distal et du canal collecteur, et leur prévalence est corrélée à la durée d'exposition au lithium. Une néphrocalcinose peut être observée.

Les maladies rénales kystiques peuvent être associées à une fréquence accrue de carcinomes à cellules claires et quelques cas de tumeurs rénales chez des patients traités par lithium ont été décrits ${ }^{[10,47]}$. Récemment, l'existence d'un risque accru de tumeurs rénales chez les patients traités par lithium a été observée ${ }^{[48]}$. Dans cette étude, le pourcentage de tumeurs rénales, en particulier de cancers et d'oncocytomes, était significativement plus élevé chez les patients traités par lithium que chez 340 patients témoins appariés sur le sexe, l'âge et le débit de filtration glomérulaire. Le ratio d'incidence standardisé de cancer rénal était également significativement plus élevé chez les patients traités par lithium que dans la population générale française : 7,51 (intervalle de confiance [IC] 95\% 1,51-21,95) et 13,69 (IC $95 \%$ 3,68-35,06) chez les hommes et femmes, respectivement.

\subsection{Physiopathologie}

La physiopathologie de la toxicité rénale du lithium est mal connue. Elle reposerait sur son accumulation dans les cellules du néphron distal et du canal collecteur via le canal ENaC. L'augmentation de la concentration de lithium dans les cellules principales engendre une inhibition de GSK3 $\beta$ et une activation de la $\beta$-caténine. II est intéressant de noter que GSK3 $\beta$ est aussi impliquée dans le maintien du cil primaire en association à la protéine suppresseur de tumeur von Hippel-Lindau ${ }^{[49]}$. En outre, le lithium affecte également la fonction de la polycystine 2 qui est l'une des protéines mutées dans la polykystose rénale autosomique dominante ${ }^{[50]}$. Ainsi, le lithium interfère avec des voies de régulation clés de la prolifération des cellules tubulaires, de la différenciation cellulaire et de l'apoptose ${ }^{[19,47,51]}$. Cela pourrait expliquer l'apparition de dilatations tubulaires, de kystes et d'une néphropathie chronique.

\subsection{Histologie}

La réalisation d'une biopsie n'est pas nécessaire, sauf en cas de doute avec un diagnostic différentiel. L'aspect histologique est celui d'une néphropathie tubulo-interstitielle chronique (Fig. 2). Des lésions de fibrose interstitielle peuvent apparaître dès 5 ans après le début du traitement ${ }^{[9]}$. II s'y associe une atrophie tubulaire, mais les glomérules sont relativement épargnés. Des kystes tubulaires peuvent être observés. Des lésions de hyalinose segmentaire et focale associées, potentiellement secondaires, ont été décrites ${ }^{[10]}$.

\subsection{Prise en charge}

Une surveillance de la lithiémie et de la fonction rénale est requise chez tous les patients traités par lithium, mais elle est parfois insuffisante. Une surveillance de la créatinine est nécessaire avant, puis tous les trois mois au cours des six premiers mois de traitement, puis une fois par an. Tout événement intercurrent susceptible de modifier la lithiémie justifie un contrôle supplémentaire de la lithiémie et de la fonction rénale. Compte tenu du risque de surdosage en lithium et de néphrotoxicité, la prescription d'anti-inflammatoires non stéroïdiens est contre-indiquée chez ces patients. La prescription de diurétiques, d'inhibiteurs 
de l'enzyme de conversion ou d'antagonistes du récepteur de l'angiotensine II est également délicate car elle expose au risque de surdosage et d'aggravation aiguë de l'insuffisance rénale. Si leur prescription est indispensable, un contrôle rapproché de la lithiémie et de la fonction rénale est nécessaire. En cas d'apparition d'une insuffisance rénale, un bilan à la recherche d'une autre cause est réalisé, puis la posologie minimale efficace est recherchée. L'objectif est d'obtenir des lithiémies proches de $0,6 \mathrm{mmol} / \mathrm{L}$ au maximum, même si l'impact de telles mesures n'a pas été démontré.

\subsection{Faut-il arrêter le traitement ?}

La question de l'arrêt du traitement est une question délicate. Le néphrologue doit prendre en compte la gravité éventuelle de la maladie psychiatrique, qui peut être associée à un risque élevé de récidive et de suicide. En effet, la mortalité attribuée à une insuffisance rénale était seulement de deux cas sur 201 comparée à celle due à un suicide après l'arrêt du traitement (42 sur 201) dans une étude reprenant la mortalité d'une cohorte de 1411 patients traités par lithium suivis dans un service de psychiatrie ${ }^{[52]}$.

La discussion de l'arrêt du traitement doit donc se faire de manière conjointe entre le patient, le néphrologue et le psychiatre. Certains patients ne souhaitent en aucun cas arrêter un traitement dont ils ont vu le bénéfice sur le plan psychiatrique, même s'il existe un risque rénal à moyen ou long terme. II faut garder à l'esprit que le bénéfice rénal de l'arrêt du traitement n'est réel que si l'insuffisance rénale est modérée (débit de filtration glomérulaire estimé supérieur à $40 \mathrm{~mL} / \mathrm{min} / 1,73 \mathrm{~m}^{2}$ ). Dans ce cas, on peut espérer une discrète amélioration, une stabilisation ou au moins une diminution de la vitesse de dégradation de la fonction rénale ${ }^{[9]}$. Si le débit de filtration glomérulaire est déjà inférieur à $40 \mathrm{~mL} / \mathrm{min} / 1,73 \mathrm{~m}^{2}$ au moment de l'arrêt du traitement, il est vraisemblable que la diminution du débit de filtration glomérulaire se poursuive à la même vitesse. Dans l'étude de Presne et al., deux tiers des patients ayant un débit de filtration glomérulaire entre 25 et $40 \mathrm{~mL} / \mathrm{min} / 1,73 \mathrm{~m}^{2}$ à l'arrêt du traitement ont continué à aggraver leur fonction rénale après l'arrêt du traitement et tous les patients qui avaient un débit de filtration glomérulaire inférieur à $25 \mathrm{~mL} / \mathrm{min} / 1,73 \mathrm{~m}^{2}$. De même, parmi les 24 patients de la cohorte de Markowitz et al., un patient s'est suicidé après l'arrêt du traitement et huit patients sur 19 ont évolué vers l'insuffisance rénale terminale malgré l'arrêt du traitement. Le meilleur prédicteur de l'évolution de la fonction rénale était la créatinine initiale ${ }^{[10]}$.

Werneke et al. ont tenté de modéliser la prise de décision du psychiatre à l'initiation du traitement, puis au cours du suivi en prenant en compte le risque suicidaire et le risque d'insuffisance rénale chronique. Pour les auteurs, à l'initiation du traitement, le risque de développer une insuffisance rénale chronique joue un rôle négligeable dans la décision d'instaurer le traitement ${ }^{[53]}$. Après 20 ans de traitement, même si certains patients ont une insuffisance rénale chronique et vont évoluer vers la dialyse, le lithium reste le traitement de choix dans $97 \%$ des cas. Le risque d'instabilité de la maladie psychiatrique est la variable la plus importante du modèle.

\section{Conduite à tenir}

La question de l'arrêt du traitement est une question délicate. Le néphrologue doit prendre en compte la gravité éventuelle de la maladie psychiatrique qui peut être associée à un risque élevé de récidive et de suicide. La discussion doit donc se faire de manière conjointe entre le patient, le néphrologue et le psychiatre. Le bénéfice rénal de l'arrêt du traitement n'est réel que si l'insuffisance rénale est modérée (débit de filtration glomérulaire supérieur à $40 \mathrm{~mL} / \mathrm{min})$. 


\section{Conclusion}

La prise prolongée de lithium est donc responsable d'un diabète insipide néphrogénique chez 40 à $50 \%$ des patients. Celui-ci est inconstamment réversible à l'arrêt, surtout si le traitement a été prolongé plus de 15 ans. De manière indépendante, une néphropathie tubulointerstitielle chronique risque de se développer après 10 à 20 ans, avec un risque d'évolution vers l'insuffisance rénale terminale. Ce risque est lié à la durée d'exposition au lithium.

\section{Points essentiels}

- La prise prolongée de lithium est responsable d'un diabète insipide néphrogénique chez 40 à $50 \%$ des patients.

- De manière indépendante, une néphropathie tubulo-interstitielle chronique peut se développer après 10 à 20 ans, avec un risque d'évolution vers l'insuffisance rénale terminale. Ce risque est lié à la durée d'exposition au lithium.

\section{Déclaration d'intérêts}

L'auteur déclare ne pas avoir de liens d'intérêts en relation avec cet article.

\section{Références}

[1] Geddes JR, Miklowitz DJ. Treatment of bipolar disorder. Lancet 2013;381:1672-82.

[2] Geddes JR, Burgess S, Hawton K, Jamison K, Goodwin GM. Long-term lithium therapy for bipolar disorder: systematic review and meta-analysis of randomized controlled trials. Am J Psychiatry 2004;161:217-22.

[3] Cipriani A, Pretty H, Hawton K, Geddes JR. Lithium in the prevention of suicidal behavior and all-cause mortality in patients with mood disorders: a systematic review of randomized trials. Am J Psychiatry 2005;162:1805-19.

[4] Trepiccione F, Christensen BM. Lithium-induced nephrogenic diabetes insipidus: new clinical and experimental findings. J Nephrol 2010;23(Suppl. 16):S43-S48.

[5] Freeman MP, Freeman SA. Lithium: clinical considerations in internal medicine. Am J Med 2006;119:478-81.

[6] Grunfeld JP, Rossier BC. Lithium nephrotoxicity revisited. Nat Rev Nephrol 2009;5:270-6.

[7] Boton R, Gaviria M, Batlle DC. Prevalence, pathogenesis, and treatment of renal dysfunction associated with chronic lithium therapy. Am J Kidney Dis 1987;10:329-45.

[8] Bendz H, Schon S, Attman PO, Aurell M. Renal failure occurs in chronic lithium treatment but is uncommon. Kidney Int 2010;77:219-24.

[9] Presne C, Fakhouri F, Noel LH, Stengel B, Even C, Kreis H. Lithium-induced nephropathy: rate of progression and prognostic factors. Kidney Int 2003;64:585-92.

[10] Markowitz GS, Radhakrishnan J, Kambham N, Valeri AM, Hines WH, D’Agati VD. Lithium nephrotoxicity: a progressive combined glomerular and tubulointerstitial nephropathy. J Am Soc Nephrol 2000;11:1439-48.

[11] Bedford JJ, Weggery S, Ellis G, McDonald FJ, Joyce PR, Leader JP. Lithium-induced nephrogenic diabetes insipidus: renal effects of amiloride. Clin J Am Soc Nephrol 2008;3:1324-31.

[12] Walker RJ, Weggery S, Bedford JJ, McDonald FJ, Ellis G, Leader JP. Lithium-induced reduction in urinary concentrating ability and urinary aquaporin 2 (AQP2) excretion in healthy volunteers. Kidney Int 2005;67:291-4. 
[13] Ljubicic D, Letica-Crepulja M, Vitezic D, Bistrovic IL, Ljubicic R. Lithium treatments: single and multiple daily dosing. Can J Psychiatry 2008;53:323-31.

[14] Bucht G, Wahlin A. Renal concentrating capacity in long-term lithium treatment and after withdrawal of lithium. Acta Med Scand 1980;207:309-14.

[15] Bendz H, Sjodin I, Aurell M. Renal function on and off lithium in patients treated with lithium for 15 years or more. A controlled, prospective lithium-withdrawal study. Nephrol Dial Transplant 1996;11:457-60.

[16] Marples D, Christensen S, Christensen EI, Ottosen PD, Nielsen S. Lithium-induced downregulation of aquaporin-2 water channel expression in rat kidney medulla. J Clin Invest 1995;95:1838-45.

[17] Kwon TH, Laursen UH, Marples D, Maunsbach AB, Knepper MA, Frokiaer J. Altered expression of renal AQPs and $\mathrm{Na}(+)$ transporters in rats with lithium-induced $\mathrm{NDI}$. Am $\mathrm{J}$ Physiol Renal Physiol 2000;279:F552-F564.

[18] Kuure S, Popsueva A, Jakobson M, Sainio K, Sariola H. Glycogen synthase kinase-3 inactivation and stabilization of beta-catenin induce nephron differentiation in isolated mouse and rat kidney mesenchymes. J Am Soc Nephrol 2007;18:1130-9.

[19] Nielsen J, Hoffert JD, Knepper MA, Agre P, Nielsen S, Fenton RA. Proteomic analysis of lithium-induced nephrogenic diabetes insipidus: mechanisms for aquaporin 2 down-regulation and cellular proliferation. Proc Natl Acad Sci USA 2008;105:3634-9.

[20] Christensen BM, Kim YH, Kwon TH, Nielsen S. Lithium treatment induces a marked proliferation of primarily principal cells in rat kidney inner medullary collecting duct. Am $\mathrm{J}$ Physiol Renal Physiol 2006;291:F39-F48.

[21] Rao R, Zhang MZ, Zhao M, Cai H, Harris RC, Breyer MD. Lithium treatment inhibits renal GSK-3 activity and promotes cyclooxygenase 2-dependent polyuria. Am J Physiol Renal Physiol 2005;288:F642-F649.

[22] Klein JD, Gunn RB, Roberts BR, Sands JM. Down-regulation of urea transporters in the renal inner medulla of lithium-fed rats. Kidney Int 2002;61:995-1002.

[23] Batlle DC, von Riotte AB, Gaviria M, Grupp M. Amelioration of polyuria by amiloride in patients receiving long-term lithium therapy. N Engl J Med 1985;312:408-14.

[24] Kishore BK, Ecelbarger CM. Lithium: a versatile tool for understanding renal physiology. Am J Physiol Renal Physiol 2013;304:F1139-F1149.

[25] Grandjean EM, Aubry JM. Lithium: updated human knowledge using an evidence-based approach: part III: clinical safety. CNS Drugs 2009;23:397-418.

[26] Christiansen C, Baastrup PC, Transbol I. Development of 'primary' hyperparathyroidism during lithium therapy: longitudinal study. Neuropsychobiology 1980;6:280-3.

[27] Bendz H, Sjodin I, Toss G, Berglund K. Hyperparathyroidism and long-term lithium therapy--a cross-sectional study and the effect of lithium withdrawal. J Intern Med 1996;240:357-65.

[28] Davis BM, Pfefferbaum A, Krutzik S, Davis KL. Lithium's effect of parathyroid hormone. Am J Psychiatry 1981;138:489-92.

[29] Hundley JC, Woodrum DT, Saunders BD, Doherty GM, Gauger PG. Revisiting lithiumassociated hyperparathyroidism in the era of intraoperative parathyroid hormone monitoring. Surgery 2005;138:1027-31 [discussion 31-2].

[30] Khandwala HM, Van Uum S. Reversible hypercalcemia and hyperparathyroidism associated with lithium therapy: case report and review of literature. Endocr Pract 2006;12:548.

[31] Awad SS, Miskulin J, Thompson N. Parathyroid adenomas versus four-gland hyperplasia as the cause of primary hyperparathyroidism in patients with prolonged lithium therapy. World J Surg 2003;27:486-8. 
[32] Dwight T, Kytola S, Teh BT, Theodosopoulos G, Richardson AL, Philips J. Genetic analysis of lithium-associated parathyroid tumors. Eur J Endocrinol 2002;146:619-27.

[33] Carchman E, Ogilvie J, Holst J, Yim J, Carty S. Appropriate surgical treatment of lithiumassociated hyperparathyroidism. World J Surg 2008;32:2195-9.

[34] Birnbaum J, Klandorf $H$, Giuliano A, Van Herle A. Lithium stimulates the release of human parathyroid hormone in vitro. J Clin Endocrinol Metab 1988;66:1187-91.

[35] Livingstone $\mathrm{C}$, Rampes $\mathrm{H}$. Lithium: a review of its metabolic adverse effects. J Psychopharmacol 2006;20:347-55.

[36] Brown EM. Lithium induces abnormal calcium-regulated PTH release in dispersed bovine parathyroid cells. J Clin Endocrinol Metab 1981;52:1046-8.

[37] Riccardi D, Gamba G. The many roles of the calcium-sensing receptor in health and disease. Arch Med Res 1999;30:436-48.

[38] Saxe A, Gibson G, Silveira E. Effects of long-term lithium infusion on normal parathyroid tissue. Surgery 1995;117:577-80.

[39] Saxe AW, Gibson G. Lithium increases tritiated thymidine uptake by abnormal human parathyroid tissue. Surgery 1991;110:1067-76 [discussion 76-7].

[40] Lepkifker E, Sverdlik A, lancu I, Ziv R, Segev S, Kotler M. Renal insufficiency in long-term lithium treatment. J Clin Psychiatry 2004;65:850-6.

[41] Bendz H, Aurell M, Balldin J, Mathe AA, Sjodin I. Kidney damage in long-term lithium patients: a cross-sectional study of patients with 15 years or more on lithium. Nephrol Dial Transplant 1994;9:1250-4.

[42] Bassilios N, Martel P, Godard V, Froissart M, Grunfeld JP, Stengel B. Monitoring of glomerular filtration rate in lithium-treated outpatients--an ambulatory laboratory database surveillance. Nephrol Dial Transplant 2008;23:562-5.

[43] Minay J, Paul R, McGarvey D, Savage G, Stevenson M, Fogarty D. Lithium usage and renal function testing in a large UK community population; a case-control study. Gen Hosp Psychiatry 2013;35:631-5.

[44] McKnight RF, Adida M, Budge K, Stockton S, Goodwin GM, Geddes JR. Lithium toxicity profile: a systematic review and meta-analysis. Lancet 2012;379:721-8.

[45] Presne C, Fakhouri F, Kenouch S, Stengel B, Kreis H, Grunfeld JP. Progressive renal failure caused by lithium nephropathy. Presse Med 2002;31:828-33.

[46] Farres MT, Ronco P, Saadoun D, Remy P, Vincent F, Khalil A. Chronic lithium nephropathy: MR imaging for diagnosis. Radiology 2003;229:570-4.

[47] Kjaersgaard G, Madsen K, Marcussen N, Christensen S, Walter S, Jensen BL. Tissue injury after lithium treatment in human and rat postnatal kidney involves glycogen synthase kinase-3beta-positive epithelium. Am J Physiol Renal Physiol 2012;302:F455-F465.

[48] Zaidan M, Stucker M, Stengel B, Vasiliu V, Hummel A, Landais P. Increased risk of solid renal tumors in lithium-treated patients. Kidney Int 2014;86:184-90.

[49] Thoma CR, Frew IJ, Krek W. The V.H.L. tumor suppressor: riding tandem with GSK3beta in primary cilium maintenance. Cell Cycle 2007;6:1809-13.

[50] Cantero M del R, Cantiello HF. Effect of lithium on the electrical properties of polycystin-2 (TRPP2). Eur Biophys J 2011;40:1029-42.

[51] Sinha D, Wang Z, Ruchalski KL, Levine JS, Krishnan S, Lieberthal W. Lithium activates the Wnt and phosphatidylinositol 3-kinase Akt signaling pathways to promote cell survival in the absence of soluble survival factors. Am J Physiol Renal Physiol 2005;288:F703-F713.

[52] Bocchetta A, Fadda D, Satta G, Del Zompo M, Gessa GL, Cocco P. Long-term lithium treatment and survival from external causes including suicide. J Clin Psychopharmacol 2007;27:544-6. 
[53] Werneke U, Ott M, Renberg ES, Taylor D, Stegmayr B. A decision analysis of long-term lithium treatment and the risk of renal failure. Acta Psychiatr Scand 2012;126:186-97.

Figure 1. Mécanisme du diabète insipide néphrogénique induit par le lithium (Li). Le lithium entre dans les cellules principales du canal collecteur par le canal sodium épithélial, ENaC, situé sur le pôle apical. Le lithium inhibe l'adénylate cyclase $(A C)$ et la génération d'adénosine monophosphate $3^{\prime}-5^{\prime}$ cyclique (AMPc). II induit une phosphorylation d'Akt, ce qui induit une inhibition de glycogène synthase kinase type $3 \beta$ (GSK3 $\beta$ ) par le biais d'une phosphorylation. Cela influe sur la régulation de l'aquaporine 2 (AQP2). Au niveau des cellules interstitielles, le lithium inhibe GSK3 $\beta$, ce qui stimule la voie cyclo-oxygénase 2-prostaglandine $E$ (COX-2$P G E)$ et engendre une régulation négative de l'AQP2 dans les cellules principales.

Figure 2. Néphropathie tubulo-interstitielle chronique sévère avec fibrose interstitielle diffuse, atrophie tubulaire et kystes tubulaires (trichrome de Masson X 40). 


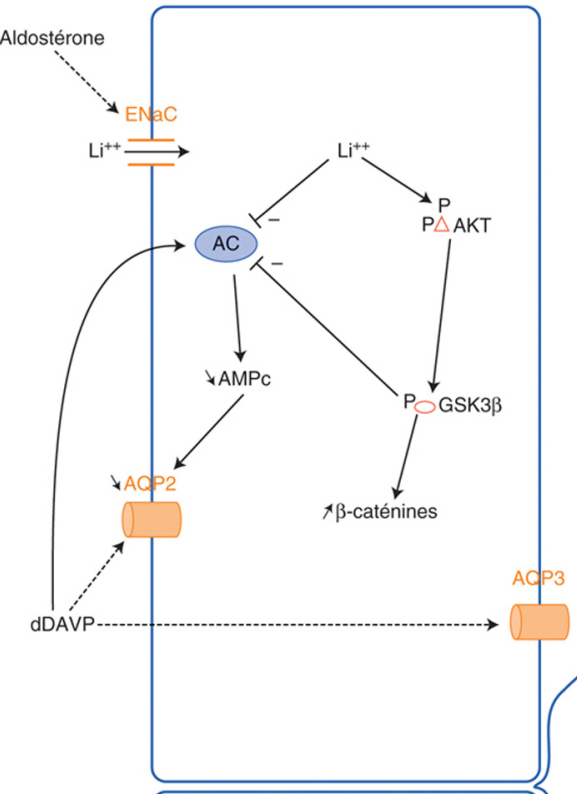

Cellule interstitielle
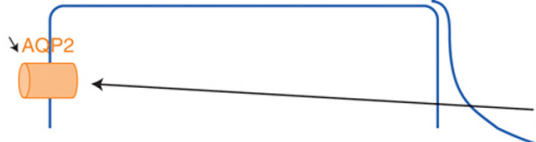


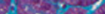

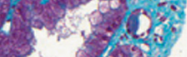
S.3.

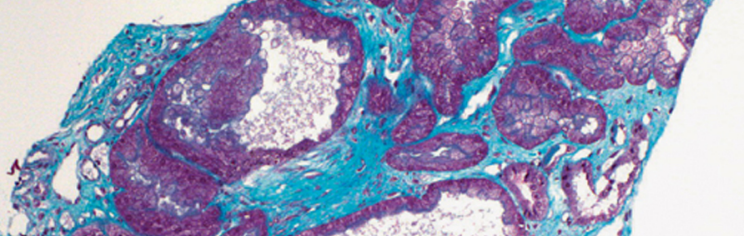

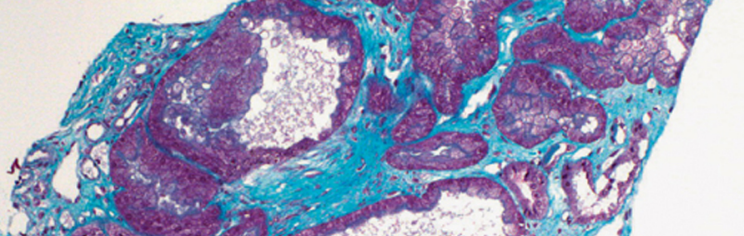

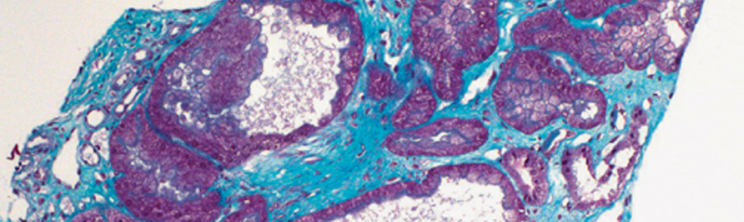

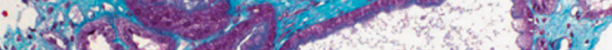

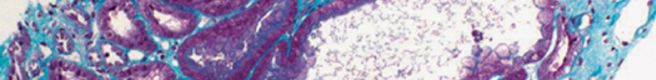
No.

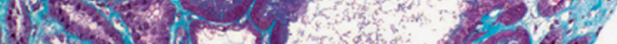
14.

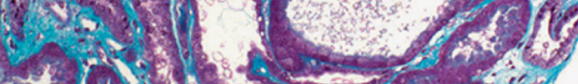

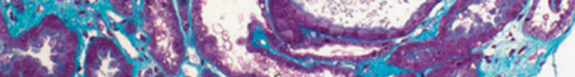

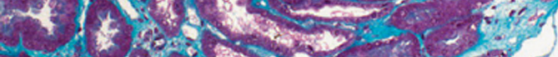

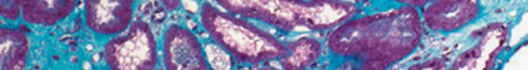

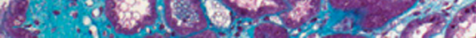

